\title{
Nous n'avons rien à cacher, au contraire!
}

\section{Christoph Bosshard}

Dr méd., Vice-président de la FMH, responsable du département Données, démographie et qualité

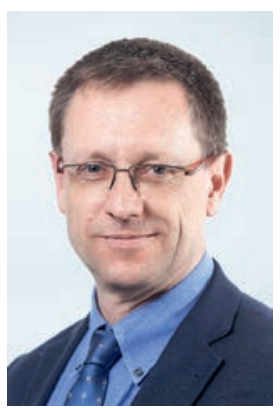

Nous y voilà de nouveau: l'Office fédéral de la statistique (OFS) démarre le 12 novembre prochain le relevé 2017 des données des cabinets médicaux et centres ambulatoires. C'est certain, personne ne se réjouit de cette sollicitation supplémentaire et la question se pose du pourquoi et de ce que cela apporte à chacun d'entre nous, à nos patients et au corps médical.

Une discussion objective, factuelle et constructive repose sur la transparence et sur des données fiables. D’après une étude du bureau BASS commandée par l'Office fédéral de la santé publique (OFSP), le revenu réel des médecins spécialistes en Suisse s'élève à 190577 francs. Un chiffre qui fait cependant l'impasse sur les revenus d'un quart du corps médical, et plus exactement des quelque 10000 médecinsassistants. L'étude ne précise pas non plus quelle part du revenu provient des primes. De plus, les chiffres avancés concernent l'année 2014, c'est-à-dire avant les réductions des tarifs par le Conseil fédéral. Avec le relevé MARS, nous avons maintenant la possibilité de procéder à des enquêtes et des analyses beaucoup plus différenciées, notamment en distinguant entre assurance obligatoire ou assurance complémentaire pour le chiffre d'affaires et en présentant de ma-

\section{L'OFS démarre le 12 novembre prochain le relevé 2017 des données des cabinets médicaux et centres ambulatoires.}

nière claire et transparente les revenus générés hors de l'activité médicale au cabinet. Grâce à cela, les résultats du relevé MAS $2015^{1}$ démontrent clairement que les chiffres produits jusqu'à présent par le corps médical sont corrects et que les coûts de fonctionnement des cabinets médicaux ne s'élèvent pas à $70 \%$ mais même à $72 \%$. Ces chiffres permettent aussi de délimiter clairement quels revenus relèvent des assurances sociales, car c'est le seul domaine qui justifie l'intérêt public pour la transparence. Que pouvait-il donc nous arriver de mieux que de voir nos affirmations confirmées et validées par la Confédération?
Pour maintenir la qualité des données au plus haut et la charge administrative au plus bas, la Caisse des médecins, NewIndex et la FMH offrent la possibilité à chaque membre d'importer ses données dans le questionnaire par un simple clic de souris. Avec la FMH, l'Office fédéral de la statistique mise sur la persuasion et renonce encore une fois cette année à des sanctions contre les membres désireux de ne pas respecter les obligations légales.

L'anonymisation des données est également garantie, et ce aussi dans la partie concernant l'autorité de sur-

\section{Que pouvait-il donc nous arriver de mieux que} de voir nos affirmations confirmées et validées par la Confédération?

veillance. En effet, les bases réglementaires concernées (ordonnance et règlement) définissent que seules des données agrégées, ne permettant pas de remonter vers les cabinets particuliers, peuvent être transmises aux destinataires (OFSP, assureurs, cantons).

Souvent, on me demande ce qui se passera avec les données si le cadre légal devait changer. Mais rappelons sans ambiguïté que ce cadre ne se laisse pas modifier aussi facilement sans raison. La collaboration constructive avec l'OFS offre ici un appui important. De plus, il est évident que les clauses concernant l'usage de ces données n'ont pas le droit d'être modifiées ultérieurement; en revanche, elles peuvent entrer en vigueur de manière proactive. En effet, tout changement des bases juridiques est immédiatement suivi de nouvelles négociations concernant notre collaboration. Cela permet de garantir que la FMH reste un partenaire actif et impliqué.

Dans ce contexte, le Comité central de la FMH recommande aux membres de participer au prochain relevé de l'OFS. Cela demande du temps mais le bénéfice est considérable. Je remercie ici tous ceux et celles qui nous soutiennent dans ce dossier. Mes remerciements s'adressent également à nos partenaires et à nos collaborateurs pour leur engagement solide et précieux. 